\title{
CONFLITOS ENTRE MACACOS-PREGO E VISITANTES NO PARQUE NACIONAL DE BRASÍLIA: POSSÍVEIS SOLUÇÕES
}

\section{Conflicts between capuchin monkeys and visitors inside the Brasília National Park: possible solutions}

Carlos Hiroo Saito

Professor, Biólogo, Doutor em Geografia (Geoprocessamento) Laboratório de Ecologia Aplicada, Departamento de Ecologia, Instituto de Biologia,

Universidade de Brasília

Brasília/DF - Brasil

carlos.h.saito@hotmail.com

Luiza Brasileiro

Bióloga, Mestre em ecologia, Programa de Pós-Graduação em Ecologia

Universidade de Brasília

Brasília/DF - Brasil

luizabrpereira@yahoo.com.br

Luzia Etelvina de Almeida

Bióloga, Especialização em Educação Ambiental

Departamento de Ecologia, Instituto de Biologia, Universidade de Brasília

Brasília/DF - Brasil

luziaetelvina@unb.br

Maria Clotilde Henriques Tavares

Professora, Doutorado em Psicologia (Neurociências e Comportamento) Centro de Primatologia, Instituto de Biologia, Universidade de Brasília Brasília/DF - Brasil mchtavares@unb.br

Artigo recebido para publicação em 03/06/2010 e aceito para publicação em 09/11/2010

RESUMO: $\quad$ Os conflitos entre os visitantes e os macacos-prego na área das piscinas do Parque Nacional de Brasília (PNB) têm origem na presença de alimentos levados pelos próprios visitantes, objetos de atração dos macacos-prego. Com base na literatura e resultados de pesquisas desenvolvidas no próprio local, o trabalho apresenta as recomendações à administração do PNB para reduzir os níveis de o conflito.

Palavras-chave: Cebus libidinosus. Macaco-prego. Visitação. Unidade de Conservação da Natureza. Parque Nacional de Brasília. Conflito. Alimentação.

ABSTRACT: The conflicts between the capuchin monkeys and the visitors inside the swimming pool areas of the Brasilia National Park (Brazil) lays on the presence of the food handled by the visitors, which attracts the monkeys. Based on the literature and on the results of researches developed in this National Park, this works presents some proposals to the National Park administration to get lower incidence of conflicts.

Keywords: Cebus libidinosus. Capuchin monkeys. Protected Areas for Conservation. Brasília National Park. Conflicts. Feeding. 


\section{INTRODUÇÃO}

Mudanças culturais na relação "homem $\mathrm{x}$ fauna silvestre" decorrentes de fatores diversos dentre os quais a redução do habitat natural e a falta de conhecimento sobre a própria fauna silvestre tem ocasionado a adoção de comportamentos inadequados do homem em relação aos animais (e.g. tentativas de alimentação, domesticação e manutenção de espécies silvestres como animais de estimação, etc). Simultaneamente, o alto grau de adaptabilidade de algumas espécies tem ocasionado interações cada vez mais estreitas e conflituosas entre humanos e animais na tentativa destes se adaptarem aos efeitos da urbanização por ação antrópica. Conflitos dessa natureza têm sido relatados pela literatura e comumente primatas têm sido vistos como uma séria ameaça e perseguidos como pragas, particularmente em situações onde eles invadem plantações (FORTHMAN-QUICK, 1986; SIEX; STRUHSAKER, 1999; HILL, 2000) ou são alimentados/provisionados por humanos (por exemplo, Semnopithecus entellus, HRDY, 1977; Macaca sylvanus, O'LEARY; FA, 1993; Macaca radiata, SCHLOTTERHAUSEN, 1998; Macaca mulatta, GUPTA, 2002).

Diversos estudos relatam a utilização por parte de espécies silvestres de áreas habitadas ou próximas a das utilizadas por humanos, bem como a utilização do lixo humano como fonte de alimento (e.g. macacos, ver BOX, 1991; coiotes, ver ELLINS, THOMPSON e SWANSON, 1983; SABBATINI et al., 2006). Dentre os primatas, macacos-prego (Cebus), graças ao fato de apresentarem uma grande flexibilidade comportamental e capacidade de aprendizagem, podem, em geral, se adaptar a uma diversidade de habitats e em certa extensão, adaptam-se à presença humana (HAWKES, HILL e O'CONNELL, 1982; SIEMERS, 2000). No entanto, interações conflituosas tem-se tornado particularmente comuns entre eles e humanos face ao aumento do contato por ocasião da urbanização e desmatamento na América do Sul (FRAGAZY et al., 2004; MENDES et al., 2005), uma vez que apresentam ampla distribuição geográfica que inclui a Bolivia, o nordeste da Argentina, as regiões do centro-oeste, nordeste e pantanal do Mato Grosso no Brasil, além do leste do Paraguai (FRAGAZY et al., 2004). Assim, macacos-prego são encontrados em praticamente em todos os tipos de florestas tropicais (JANSON, 1985), em florestas secundárias e em fragmentos limitados de floresta (HAWKES, HILL e O'CONNELL, 1982).

No Distrito Federal, o Parque Nacional de Brasília (PNB), é um dos locais em que são encontrados grupos naturais de macacos-prego (Cebus libidinosus) em contato com seres humanos.

A espécie Cebus libidinosus é considerada uma subespécie do Cebus apella, pertencente à família Cebidae (GROVES, 2001) e vive em grupos que variam de 3-5 até 30-40 indivíduos (FREESE; OPPENHEIMER, 1981) nos quais tipicamente são encontrados um macho e uma fêmea dominantes, além de outros machos e fêmeas e vários outros juvenis e filhotes. Sua dieta é onívora (OPPENHEIMER, 1968) e baseada principalmente em frutos, embora também façam parte dela flores, brotos, raízes, pequenos anfíbios, répteis, artrópodes, ovos, filhotes de pássaros e pequenos mamíferos (FRAGASZY, VISALBERGHI e FEDIGAN, 2004). Como outros primatas, eles apresentam estratégias para modificar a sua dieta em função da baixa disponibilidade de alimentos, o que inclui o consumo de recursos alternativos e a divisão do grupo em grupos menores, com vistas ao aumento do consumo e a redução do gasto energético (PERES, 1991; TERBORGH, 1983; CLUTTON-BROCK, 1977). Em reposta à disponibilidade de novas fontes alimentares e à disponibilidade sazonal de recursos, são modificados também os padrões de atividade da espécie (SABBATINI et al., 2008).

O PNB, criado pelo Decreto no 241, de 29 de novembro de 1961, é uma Unidade de Conservação da Natureza de Proteção Integral, consagrada como Zona Núcleo da Reserva da Biosfera do Cerrado pela Unesco. Considerado um parque nacional urbano, recebe um afluxo grande de visitantes para lazer, sobretudo pela presença de piscinas de água mineral na sua área de uso intensivo. Esse atrativo tem levado à presença de cerca de 300 mil visitantes/banhistas por ano (ABDALA, 2002) à área das piscinas, o que intensifica o contato entre os visitantes e os macacos-prego e propicia o surgimento de conflitos, com prejuízos mútuos.

Diante do agravamento desse quadro, a direção do PNB convocou no ano de 2006, pesquisadores 
da Universidade de Brasília para apresentar propostas visando a redução dos conflitos. Nesse sentido, foram resgatados resultados de estudos realizados anteriormente no referido Parque que norteiam as propostas elaboradas e apresentadas a seguir.

No sentido de verificar como ocorria essa interação entre macacos e visitantes no $\mathrm{PNB}$, foi realizado um estudo entre agosto de 2003 e abril de 2004 (SABBATINI et al., 2006). Este estudo foi dividido basicamente em duas partes: entrevista com os visitantes e observação das interações, onde foram observados 380 episódios de interações entre macacos-prego e humanos na área da piscina velha e 529 episódios de alimentação dos macacos a partir de restos de alimentos consumidos pelos humanos, deixados nas lixeiras, ou no chão do Parque. As entrevistas foram realizadas com 300 visitantes que freqüentavam o Parque em dois períodos durante a semana (início da manhã 6:30 h às 10:00 h, horário de almoço 12:00 h às 14:00 h) e aos finais de semana (10:00 h às 16:00 h) no sentido de verificar as suas atitudes em relação aos macacos e o seu grau de conhecimento sobre a espécie.

Foram avaliados no estudo fatores como classe sexo-etária dos macacos e humanos, a distância mínima entre ambos durante as interações, a densidade de pessoas na área onde ocorriam as interações, se as mesmas envolviam a presença de alimento, se ocorria a obtenção ou não do alimento pelos macacos e se estes apresentavam comportamentos e/ou posturas agressivas durante as interações.

Os resultados indicaram que: 1) praticamente $80 \%$ das interações entre os macacos e humanos envolveram a presença de comida; 2) a maior parte dessas interações foi iniciada pelos visitantes $(47,3 \%)$ que em $56 \%$ dos casos forneceram espontânea e diretamente alimentos para os animais, atiraram-nos para que estes pudessem apanhá-los ou ainda deixaram restos de alimento a fim de que os animais pudessem ter acesso; 4) em apenas 19,5\% dos casos, os macacos roubaram alimentos dos visitantes; 5) a distância mínima ente os visitantes e os macacos variou de $1 \mathrm{~cm}$ a $1 \mathrm{~m}$; 6) humanos adultos e macacos machos adultos foram os que mais interagiram. Mulheres e crianças, bem como macacas adultas, jovens e filhotes apresentaram baixo número de interações; 7) $63,9 \%$ dos visitantes do PNB desconheciam o nome popular da espécie de macaco que freqüenta a área da piscina e a maioria dos entrevistados desconhecia a sua dieta; 8) $70,9 \%$ dos visitantes apresentaram atitudes positivas em relação aos macacos-prego.

\section{OS PREJUÍZOS AOS MACACOS-PREGO}

A exemplo do que vem ocorrendo em outros locais, os macacos-prego (Cebus libidinosus) do Parque Nacional de Brasília que freqüentam a área de visitação encontram-se em competição direta com humanos, têm fácil acesso a alimentos antropogênicos e alimentam-se do lixo produzido pelos visitantes.

Cerca de $60 \%$ da dieta desses macacos-prego é de alimentos antropogênicos, os quais são levados pelos visitantes ou vendidos nas lanchonetes (PINHA et al., 2004). A ingestão desse tipo de alimento pode causar sérios problemas de saúde nos macacos, além de alterações comportamentais (FORTHMAN-QUICK; DEMMENT, 1988; GUERRERA et al., 2002; SAJ, SICOTTE e PATERSON, 1999; SABBATINI, et al. 2006).

Pelo fato de a maior parte dos alimentos antropogênicos consumidos pelos macacos apresentar um alto nível calórico, a ingestão desses alimentos pode aumentar os níveis de glicose e colesterol, causando obesidade, problemas cardíacos e diabetes. A ingestão de grande quantidade de açúcar pode provocar também o aparecimento de cáries.

A substituição da dieta natural por alimentos inadequados para seu consumo e com baixo potencial nutritivo pode causar déficit nutricional aos macacos, e a ingestão de itens antropogênicos adulterados ou em baixo estado de conservação que são encontrados no lixo pode levar não apenas a alterações da função gastrintestinal, mas também ao comprometimento do sistema imunológico e do estado de saúde dos animais de modo geral (FORTHMAN-QUICK; DEMMENT, 1988, SABBATINI et al., 2006).

Além dos prejuízos já citados para os animais do ponto de vista nutricional, devemos acrescentar outros fatores negativos, tais como o estabelecimento de uma relação de dependência dos macacos em direção aos humanos no sentido de obtenção de alimento, assim como a redução dos comportamentos de medo que 
a espécie naturalmente apresenta quando mantida no ambiente silvestre sem o contato intenso com humano.

Tendo em vista todos esses possíveis efeitos maléficos da alteração da dieta para os macacos-prego, o fornecimento de alimentos a esses animais pode ser considerado por alguns como um ato de mau-trato, que está sujeito a sanções penais e administrativas de acordo com o artigo 32 da Lei de Crimes Ambientais (Lei n ${ }^{\circ} 9.605$ de fevereiro de 1998).

Pode-se ainda apontar a produção de impacto negativo sobre a rede de interdependências no ecossistema, decorrente dessa alteração da dieta dos macacosprego: a ingestão de alimentos antropogênicos provoca em potencial, uma diminuição da ingestão de frutos do Cerrado e, conseqüentemente, uma redução da função ecológica desses animais, como importantes agentes dispersores de sementes, além de provocar degradação do ambiente e prejuízo também a outros integrantes da fauna silvestre, visto que já foi encontrado lixo industrializado, muito provavelmente levado pelos macacos, (por exemplo, plástico de salgadinhos) até cerca de $30 \mathrm{~m}$ adentro da mata.

No estudo conduzido por Sabbatini et al. (2006) foi constatado que os visitantes têm o hábito de oferecer alimentos aos macacos-prego, embora não admitam publicamente este comportamento. Adicionalmente, a exemplo do que já verificado para outras espécies de primatas (e.g. FORTHMAN QUICK, 1986; LEE et al., 1986; O'LEARY; FA, 1993; SAJ, SICOTTE e PATERSON, 1999), Sabbatini et al. (2008) observaram em estudo realizado no PNB que os macacos modificam os padrões de busca por alimento, reduzem a sua área de uso e diminuem o tempo gasto no forrageamento na presença dos visitantes quando a chance de obtenção de alimentos aumenta. Os resultados indicaram também que o grupo despendeu menos tempo forrageando itens naturais do que outros grupos de macacos-prego vivendo em habitats similares. Outro aspecto importante foi o fato de que o comportamento dos animais foi diretamente afetado pelo número de visitantes do Parque, ou seja, eles gastaram mais tempo na floresta e alimentaram-se em maior proporção de recursos naturais quando a presença humana era escassa ao invés do que ocorreu quando a presença dos visitantes era maciça.
Em outro estudo (processo 02063000065/200640, licença 009/06), realizado com visitantes no ano de 2006, constatou-se que os visitantes incluem como alimentos aos mesmos animais diversos itens antropogênicos, sugerindo que estes visitantes consideram que seria benéfico e viável oferecer alimentos à fauna silvestre . Neste estudo, somente $29 \%$ dos entrevistados posicionaram-se contrariamente ao oferecimento de qualquer tipo de alimento aos primatas e os demais respondentes concordam com o oferecimento de algum tipo de alimento antropogênico pelos visitantes, principalmente frutas. Esta atitude tem sido apontada pelos gestores do PNB como um dos principais motivos para a ocorrência de conflitos.

Cabe salientar que esses conflitos entre humanos e macacos-prego favorecem a constituição de um imaginário social em que esses animais passam a ser vistos como agressores dos freqüentadores do PNB e, portanto, indesejáveis naquela localidade, sobretudo devido à visão distorcida que os visitantes têm do PNB, por meio da qual consideram-no como uma simples área de lazer, e não como uma Unidade de Conservação da Natureza.

\section{OS PREJUÍZOS AOS VISITANTES}

De acordo com a Lei n ${ }^{\circ} 9.985$ de 2000, o Parque Nacional tem como objetivo a preservação de ecossistemas naturais de grande relevância ecológica. A recreação em contanto com a natureza e o turismo ecológico devem ser permitidos com o intuito de desenvolver atividades de educação e interpretação ambiental. No entanto, as interações entre os visitantes do Parque e os macacosprego vêm, gradualmente, tornando-se conflituosas, o que tem comprometido o bem-estar dos primeiros em relação ao usufruto de suas atividades de recreação e lazer junto à natureza. Prejuízos diversos como a perda de objetos pessoais, alimentos furtados pelos macacosprego e lesões corporais provocadas por mordidas dos animais têm sido relatados.

Como mencionado por Fragazy et al. (2004), é importante ressaltar que mordidas dos macacos-prego podem levar à transmissão de doenças como a raiva e herpes para os visitantes, o que agrava a situação de conflito, podendo, a longo prazo, criar um clima de medo e insegurança, ou mesmo de aversão a esses animais. 
Sabbatini et al., (2006) verificaram que a maior parte dos visitantes do PNB (70,9\%) gosta dos primatas ou tem atitudes positivas em relação aos mesmos. Ademais, 63,2\% dos visitantes atribuem características positivas a esses animais. Esses resultados indicam que a presença de macacos-prego no PNB é importante para a recreação dos visitantes em contato com a natureza, o que pode, inclusive, estimular a visitação. No entanto, é importante retomar as informações anteriormente apresentadas de que os visitantes têm por hábito oferecer alimentos aos animais, embora não admitam publicamente este comportamento (SABBATINI et al., 2006) e de que $71 \%$ dos entrevistados de pesquisa recente concordam com o oferecimento de algum tipo de alimento antropogênico pelos visitantes. Isto indica que o sentimento de gostar dos animais vem acompanhado de atitudes e comportamentos que favorecem o desencadeamento de conflitos entre ambos, com conseqüências preocupantes também para os humanos.

Segundo relato da Administração do Parque Nacional de Brasília, os conflitos vêm se intensificando, motivados pela obtenção fácil de alimentos pelos macacos-prego, e no estágio atual, mesmo quando não há oferecimento de alimentos pelos visitantes, os primatas assediam os visitantes na tentativa de obtenção do alimento, algumas vezes podendo, inclusive, tomar à força os alimentos de crianças. Embora o número de acidentes ou conflitos não tenha sido registrado enquanto ocorrências formais e demandem, portanto, um levantamento sistemático, inclusive sobre a percepção do conflito pelos funcionários, a indicação de frequências semanais de conflitos relatada verbalmente e a descrição da conduta dos assédios/ataques pelos animais sugerem que o conflito não seria evitado com a simples conscientização dos visitantes no sentido de não mais oferecer alimentos aos macacos-prego. Isto porque a situação relatada indica um nível de dependência dos animais ao alimento antropogênico que configura não apenas a aceitação do alimento de forma ocasional quando este é fornecido pelos visitantes, mas o desenvolvimento de uma atitude pró-ativa de busca e obtenção do alimento por parte dos primatas.

$\mathrm{O}$ agravamento dos conflitos alcançando a situação de ocorrência de mordidas pode gerar sérios prejuízos, sobretudo em crianças, particularmente se estas mordidas vierem a ser realizadas por macacos machos adultos. Apesar do estudo (processo 02063000065/2006-40, licença 009/06) realizado no próprio Parque Nacional de Brasília ter constatado que mais de $60 \%$ dos entrevistados classificaram corretamente as expressões dos macacos-pregos, ou seja, foram capazes de reconhecer um estado de agressividade dos mesmos, o mesmo nível de reconhecimento pode não se dar com as crianças, sobretudo se estiverem concentradas no ato de oferecer alimentos aos primatas, ou brincar de oferecer e retirar os alimentos, o que pode acentuar a irritabilidade nos animais. Mesmo entre os adultos entrevistados, deve-se ressaltar que o reconhecimento correto da expressão facial não se deu para quase $40 \%$, o que é um número elevado em se tratando de risco de acidentes.

Este mesmo estudo apontou a opinião dos entrevistados sobre a responsabilidade institucional do PNB enquanto Unidade de Conservação da Natureza o que, de alguma forma, poderia contribuir para o agravamento dos conflitos relatados. Nesse sentido, merecem reflexão por parte da administração desta UC os seguintes aspectos:

Falta de informações fornecidas aos visitantes sobre a presença dos macacos-prego e outros animais silvestres na área do Parque Nacional. Falta de sinalização ambiental em quantidade e qualidade apropriada nas dependências do PNB sobre a proibição de alimentação dos animais silvestres.

Falta de informações fornecidas aos visitantes sobre como lidar com os animais, especialmente com os macacos-prego, em virtude da possibilidade e proximidade de contato em casos de conflito.

Atuações tímidas ou mesmo ausência de atuação por parte dos funcionários do PNB observadas no sentido de coibir atos de alimentação dos animais por parte dos humanos, bem como educar os visitantes.

Dificuldade de controle do comportamento humano em virtude do grande fluxo de visitantes permitido pelo Parque, sobretudo aos finais de semana e feriados, bem como do baixo número de agentes ambientais devidamente treinados para a tarefa. 
Ausência de conseqüências (penalidade, multa, impossibilidade de comparecimento posterior, etc) para os visitantes que são flagrados alimentando os animais como forma de educação ambiental.

\section{POSSÍVEIS PROPOSTAS PARA SOLUÇÃO DO CONFLITO}

Com base no exposto, reiterando o fato de que os macacos-prego buscam ativamente obter o alimento portado pelos visitantes, mesmo quando estes não os oferecem, conclui-se que a simples conscientização dos visitantes para não mais oferecer alimentos a esses animais seria relativamente pouco eficaz, a curto prazo. Qualquer expectativa de resultados positivos por meio da educação ambiental nessa situação demandaria um conjunto de ações articuladas, de caráter educacional, midiático e de infra-estrutura, que demandariam tempo e recursos. Portanto, tendo em vista a necessidade de buscar soluções de curto prazo, a indicação que se apresenta é direcionada para se coibir a presença de alimentos na área de visitação pública do $\mathrm{PNB}$, de forma que os macacos-prego não encontrem motivos para aproximação e, por conseguinte, geração de conflitos com os visitantes.

Para coibir a presença de alimentos seria recomendável que:

A direção do PNB proibisse o ingresso de visitantes portando alimentos e bebidas. Para isto, os visitantes deveriam ser previamente informados, com antecedência, da data de entrada em vigor da proibição, e tal fato deveria ser amplamente divulgado nos meios de comunicação de massa da cidade. Esta iniciativa de comunicação antecipada teria por objetivo a maior conscientização dos visitantes, de forma a minimizar o impacto negativo e eventual resistência ao cumprimento da norma pelos visitantes, fato já detectado na pesquisa conduzida em 2006 (processo 02063000065/200640, licença 009/06).

Essa medida estaria de acordo com os objetivos específicos da Unidade de Conservação constante do seu Plano de Manejo, que é proteger as espécies da fauna e proporcionar oportunidades para recreação e atividades de lazer orientadas para o conhecimento e apreciação dos recursos naturais preservados no $\mathrm{PNB}$, ao mesmo tempo divulgando juntamente aos visitantes a contribuição do Parque para a qualidade de vida da população. No mesmo documento, especificamente para as zonas de uso intensivo, o plano de manejo normatiza que estas devem oferecer áreas e infra-estrutura próprias para recreação e atividades de lazer, interpretação e educação ambiental, de modo a não causar danos indiscriminados aos ambientes do PNB e que, para tanto, não seriam permitidas atividades que não estivessem em consonância com os objetivos do Parque. Os pontos de venda de alimentos (lanchonetes) na área das piscinas deveriam ser fechados, podendo ser transferidos para a área externa ao PNB. No caso em que a remoção configure-se como inviável, deve-se providenciar o cercamento total (nas laterais e na parte superior) da área ao redor do estabelecimento, de forma que a comercialização de alimentos e o seu consumo pelos visitantes ficassem restritos ao interior da área cercada. Isto seria fundamental, do contrário, os animais se deslocariam para outras áreas de alimentação e o problema persistiria, a exemplo do que já foi demonstrado em outros estudos (LEE et al., 1986)

Estas medidas apresentam as seguintes vantagens:

O provável declínio a curto prazo dos conflitos. Permitiria ainda preservar a imagem do PNB junto aos visitantes, no que tange à maior segurança principalmente das crianças, que segundo os funcionários do PNB, tem sido os maiores alvos de mordidas.

A busca de solução para o acesso às lixeiras pelos macacos-prego, uma vez que estas deixariam de servir como receptáculo de sobras de alimento, visto que não haveria mais presença de sobras de alimentos nestes recipientes. Isto também beneficiaria outros 
animais que também já foram vistos buscando alimentos nessas lixeiras, como anta (Tapirus terrestris), cutia (Dasyprocta aguti) e várias espécies de aves.

A diminuição da quantidade de lixo encontrada na mata, nociva ao ecossistema, de modo geral, que também polui visualmente $\mathrm{o}$ ambiente do PNB.

A economia na busca de recursos para desenvolver trabalhos de fiscalização nas piscinas e conscientização dos visitantes para não oferecer alimentos aos animais. Além disso, os esforços em termos de investimento humano e de recursos financeiros em educação ambiental poderiam ser canalizados para outras atividades desta natureza, tais como trilhas, sensibilização e valorização da natureza como um todo e do próprio PNB.

A adoção de uma atitude pró-ativa da direção do PNB. Nestes termos, evitaria que os visitantes que sejam alvo de agressões dos macacos-prego venham a responsabilizar judicialmente o PNB por negligência ou omissão frente ao problema.

Possivelmente, o fim dos conflitos evitaria também que os visitantes criassem uma imagem negativa dos macacos, permitindo uma maior aproximação do homem com a natureza, e a convivência harmônica entre estes e animais, convergindo para os objetivos do Parque Nacional. Outro aspecto importante seria que a presença dos macacos-prego poderiam estimular a visitação, uma vez que a maior parte das pessoas que freqüentam o PNB tem atitudes positivas em relação aos animais.

Considerando que o poder público tem a incumbência de proteger a fauna e impedir práticas que coloquem em risco a função ecológica das espécies, de acordo com a Constituição Federal de 1988, torna-se necessário que o órgão ambiental responsável pela administração do PNB implemente medidas para evitar a perda da função ecológica dessa espécie.

O Instituto Brasileiro do Meio Ambiente e dos Recursos Naturais Renováveis - IBAMA e o Instututo Chico Mendes de Conservação da Bio- diversidade - ICMBio, têm como incumbência, a proteção de ecossistemas e espécies. A omissão da autoridade ambiental competente diante desses conflitos é considerada como infração administrativa ambiental, de acordo com a lei de crimes ambientais (lei 9.605, de 1998). Portanto, é imprescindível que o órgão ambiental responsável pela administração do PNB atue, urgentemente, para evitar o desequilíbrio do ecossistema natural do parque e assegurar o bemestar dos visitantes.

Acreditamos com essas propostas, contribuir para o melhor cumprimento do papel do PNB enquanto Unidade de Conservação da Natureza, na medida em que implemente meios para evitar a perda da função ecológica dessa espécie ao mesmo tempo em que conserve a biodiversidade, assegure o bemestar dos visitantes e favoreça o contato harmônico e a aproximação de seres humanos com a natureza.

\section{REFERÊNCIAS}

ABDALA, G. C. Uma abordagem socioecológica do Parque Nacional de Brasília - estudo de caso. Brasília: UNESCO, 2002.

BOX, H. O. (Ed.) Primate responses to environmental change. London: Chapman \& Hall.1991.

CLUTTON-BROCK, T. Some aspects of intraspecific variation in feeding and ranging behavior in primates. In:__ (Ed.), Primate Ecology, Academic Press, New York. 1977. p. 539-556.

ELLINS, R.; THOMPSON, L.; SWANSON, W. E. Effects of novelty and familiarity on ilness-induced aversions to food and place cues in coyotes (Canis latrans). Journal of Comparative Psychology, n.97, p. 302-309, 1983.

FORTHMAN-QUICK, D. L. Activity budgets and the consumption of human food in two troops of baboons, Papio anubis, at Gilgil, Kenya. In: ELSE, J. G., LEE, P. C. (Ed.). Primate Ecology and Conservation. Cambridge-UK: Cambridge University Press, 1986. p. 221-228. 
FORTHMAN-QUICK, D. L.; DEMMENT, M. W. Dynamics of exploitation: Differential energetic adaptations of two troops of baboons to recent human contact. In: FA, J. E.; SOUTHWICK, C. H. (Ed.). Ecology and Behavior of Food-Enhanced Primate Groups, New York: Alan R. Liss, Inc., 1988. p. 25-51.

FRAGASZY, D. M.; VISALBERGHI, E.; FEDIGAN, L. M. The complete capuchin: the biology of the genus Cebus. 1. ed. Cambridge-UK: Cambridge University Press, 2004.

FREESE, C. H.;OPPENHEIMER, J. R. The Capuchin Monkeys, Genus Cebus. In: COIMBRA-FILHO, A. F.; MITTERMEIER, R. A.(Ed.), Ecology and Behavior of Neotropical Primates, Rio de Janeiro, Academia Brasileira de Ciências. 1981. p. 331-339.

GUERRERA, W.; SLEEMAN, J. M.; JASPER, S. B.; PACE, L. B.; ICHINOSE, T. Y.; REIF, J. S. Medical Survey of the Local Human Population to Determine Possible Health Risks to the Mountain Gorillas of Bwindi Impenetrable Forest National Park, Uganda. International Journal of Primatology. n.24, p. 197207, 2002.

GROVES, C. P. Primate Taxonomy. Washington, D.C.: Smithsonian Institution Press. 2001.

GUPTA, A. K. Is Hindu religion responsible for man-monkey conflict? In: CARING FOR PRIMATES - CONGRESS OF THE INTERNATIONAL PRIMATOLOGICAL SOCIETY, 19, 2002, Beijing. Resumos... Beijing, China, p. 176.

HAWKES, K., HILL, K.; O'CONNELL, J. F. Why hunters gather: optimal foraging and the Achè of eastern Paraguay. Am Ethnol, v.9, p. 379-398, 1982.

HILL, C. M. Conflict of interest between people and baboons: crop raiding in Uganda. Int. J. Primatol. v.21, n.2, p. 299-315, 2000.

HRDY, S. B. The Langurs of Abu. Cambridge: Harvard University Press, 1977.
JANSON, C.H. Aggressive competition and individual food consumption in wild brown capuchin monkeys (Cebus apella). Behav. Ecol. Sociobiol., v. 18, p.125$138,1985$.

LEE, P. C.; BRENNAN, E. J.; ELSE, J. G.;ALTMANN, J. Ecology and behaviour of vervet monkeys in a tourist lodge habitat. In: ELSE, J. G., LEE, P. C. (Ed.), Primate Ecology and Conservation. Cambridge-UK: Cambridge University Press, 1986. p. 229-235.

MENDES, F. D. C., ROCHA, S. A. A., BALESTRA, R., GUIMARÃES, Z. F. S.; PORTELA, R. C. Padrões comportamentais de Cebus apella libidinosus em fragmentos de matas urbanas: adaptabilidade, problemas de convivência e manejo. In: DESAFIOS PARAA CONSERVAÇÃO EM PAISAGENS FRAGMENTADAS - CONGRESSO BRASILEIRO DE PRIMATOLOGIA, 11, 2005, Porto Alegre. Resumos... Porto Alegre, Brasil. p. 39.

O'LEARY, H.; FA, J. E. Effects of tourists on barbary macaques at Gibraltar. Folia Primatol., n. 61, p. 7791, 1993.

PERES, C. A. Seed predation of cariniana micrantha (lecythidaceae) by brown capuchin monkeys in Central Amazonia. Biotropica, vol. 23, n. 3, p. 262-270, 1991.

PINHA, P. S.; WAGA I. C.; SABBATINI G.; TAVARES M. C. H. Comportamento alimentar de um grupo de macacos-prego Cebus apella (Cebidae, Primates) no Parque Nacional de Brasília. In: ENCONTRO ANUAL DE ETOLOGIA, 22, 2004, Campo Grande. Anais... Campo Grande: SBEt, UFMS.

SABBATINI, G.; STAMMATI, M.; TAVARES, M. C. H.; GIULIANI, V.; VISALVERGHI, E. Interactions between humans and capuchin monkeys (Cebus libidinosus) in the Parque Nacional de Brasília, Brazil. Applied Animal Behaviour Science, n. 97, p.272-283, 2006.

SABBATINI, G.; STAMMATI, M.; TAVARES, M. C. H.; GIULIANI, V.; VISALVERGHI, E. Behavioral 
flexibility of a group of bearded capuchin monkeys (Cebus libidinosus) in the National Park of Brasília (Brazil): consequences of cohabitation with visitors. Braz. J. Biol., v. 68, n. 4, p. 685-693, 2008.

SAJ, T.; SICOTTE, P.; PATERSON, J. D. Influence of human food consumption on the time budget of vervets. Int. J. Primatol., v. 20, n. 6, p. 977-994, 1999.

SCHLOTTERHAUSEN, L., Poor creatures! They can't find food in the forest: people's views on 10 monkeys in South India. In: Congress of the International Primatological Society, 17., 1998, Antananarivo. Resumos... Antananarivo, Madagascar: University of Antananarivo.

SIEMERS, B. M. Seasonal variation in food resource and forest strata use by brown capuchin monkeys (Cebus apella) in a disturbed forest fragment. Folia Primatol., n. 71, p. 81-184, 2000.

SIEX, K. S.; STRUHSAKER, T. T. Colobus monkey and coconuts: a study of perceived human-wildlife conflicts. J. Appl. Ecol., n. 36, p. 1009-1020, 1999.

TERBORGH, J. Five New World Primates: a Study in Comparative Ecology. New Jersey: Princeton University Press. 1983. 